TAIWANESE JOURNAL OF MATHEMATICS

Vol. 12, No. 4, pp. 873-886, July 2008

This paper is available online at http://www.tjm.nsysu.edu.tw/

\title{
MINIMAX DEGREES OF QUASIPLANAR GRAPHS WITH NO SHORT CYCLES OTHER THAN TRIANGLES
}

\author{
Oleg V. Borodin ${ }^{1}$, Anna O. Ivanova ${ }^{1}$, \\ Alexandr V. Kostochka ${ }^{2}$ and Naeem N. Sheikh \\ Dedicated to Professor Ko-Wei Lih on the occasion of his 60th birthday.
}

\begin{abstract}
For an edge $x y$, let $M(x y)$ be the maximum of the degrees of $x$ and $y$. The minimax degree (or $M$-degree) of a graph $G$ is $M^{*}(G)=$ $\min \{M(x y) \mid x y \in E(G)\}$. In order to get upper bounds on the game chromatic number of planar graphs, He, Hou, Lih, Shao, Wang, and Zhu showed that every planar graph $G$ without leaves and 4-cycles has minimax degree at most 8 , which was improved by Borodin, Kostochka, Sheikh, and Yu to the sharp bound 7 . We show that every planar graph $G$ without leaves and 4and 5 -cycles has $M$-degree at most 5 , which bound is sharp. We also show that every planar graph $G$ without leaves and cycles of length from 4 to 7 has $M$-degree at most 4 , which bound is attained even on planar graphs with no cycles of length from 4 to arbitrarily large number. Besides, we give sufficient conditions for a planar graph to have $M$-degrees 3 and 2 . Similar results are obtained for graphs embeddable into the projective plane, the torus and the Klein bottle.
\end{abstract}

\section{INTRODUCTION}

By quasiplanar graphs we mean those embeddable in the surfaces with nonnegative Euler characteristics; i.e., the plane, the projective plane, the torus and the Klein bottle. For brevity, we will call a graph embeddable into the projective plane a projective-planar graph, embeddable into the torus a toroidal graph, and embeddable into the Klein bottle a Kleinian graph. Recall that every planar graph is also projective-planar. For an edge $x y$ in a graph $G$, the maximum degree $M(x y)$ is the

Received December 4, 2007, Accepted February 27, 2008.

Communicated by Gerard J. Chang.

2000 Mathematics Subject Classification: 05C15.

Key words and phrases: Planar graphs, Decomposition, Short cycles.

${ }^{1}$ Research supported in part by RFBR grants 05-01-00816 and 06-01-00694.

${ }^{2}$ Research supported in part by NSF grant DMS-0650784 and RFBR grant 08-01-00673. 
maximum of the degrees of $x$ and $y$. The minimax degree (for short, $M$-degree) of a graph $G$ is $M^{*}(G)=\min \{M(x y) \mid x y \in E(G)\}$. Let $\delta(G)$ and $\Delta(G)$ denote the minimum and maximum degrees of a graph $G$, respectively.

Wernicke [17] proved that $M^{*}(G) \leq 6$ for every planar graph $G$ with $\delta(G)=5$. Kotzig [15] proved that $M^{*}(G) \leq 7$ for every planar graph $G$ with $\delta(G) \geq 4$. Borodin [4] showed that $M^{*}(G) \leq 10$ for every planar graph $G$ with $\delta(G) \geq 3$, extending Kotzig's similar result [14] on 3-polytopes. (This last upper bound was conjectured by P. Erdos and announced by D. Barnette (see Grünbaum [12], p. 454) to be true but seems to have never been published.) All these bounds are tight.

The $M$-degree of planar graphs with $\delta(G) \geq 2$ is not bounded from above. For example, $M^{*}\left(K_{2, n}\right)=n$. Note that every cycle in $K_{2, n}$ has length four. He, Hou, Lih, Shao, Wang and Zhu [13] found upper bounds on $M$-degrees of planar graphs with $\delta(G) \geq 2$ and restrictions on girth. They proved the following results.

Theorem 1. (Corollary 2.1 in [13]). Let $G$ be a connected planar graph with $\delta(G) \geq 2$. Then

$$
M^{*}(G) \leq \begin{cases}5, & \text { if } g(G) \geq 5 \\ 3, & \text { if } g(G) \geq 7 \\ 2, & \text { if } g(G) \geq 11\end{cases}
$$

Theorem 2. (Corollary 2.1 in [13]). Let $G$ be a connected planar graph with $\delta(G) \geq 2$. If $G$ does not contain 4 -cycles, then $M^{*}(G) \leq 8$.

He et al. used these bounds to estimate from above the game chromatic number (introduced by Bodlaender [1]) and the game coloring number of the corresponding graphs. Note that the game chromatic number is never greater than the game coloring number. They used the result in [11] that the game coloring number of a tree is at most 4 and the fact that if the edges of a graph $G$ are partitioned into a tree and a graph $H$, then the game coloring number of $G$ is at most $4+\Delta(H)$. In particular, Theorem 2 yields that every quadrangle-free planar graph can be decomposed into a forest and a graph with maximum degree at most 7 , and therefore the game chromatic number and the game coloring number of every such graph $G$ are at most 11 . The bounds in Theorem 1 are tight. It is also mentioned in [13] that it is not known whether 8 in Theorem 2 is the exact bound.

Borodin, Kostochka, Sheikh, and $\mathrm{Yu}$ [9] proved that the $M$-degree of any quadrangle-free graph $G$ with $\delta(G) \geq 2$ is at most 7 if $G$ is projective-planar, and is at most 8 if $G$ is toroidal and Kleinian. Both bounds are tight.

An induced cycle $C=v_{1} v_{2} \ldots v_{2 k}$ in a graph $G$ is called 2-alternating if $d\left(v_{1}\right)=d\left(v_{3}\right)=\ldots=d\left(v_{2 k-1}\right)=2$. This notion, introduced in [3], as well as its subsequent variations, turned out to be useful in some coloring and edgedecomposition problems on quasiplanar graphs (see, for example, $[4,10,6,7,13$, 
$16,18])$.

Suppose $G$ is a planar graph with $\delta(G) \geq 2$, and instead of forbidding all 4-cycles we forbid only 2-alternating 4-cycles. Then $M^{*}(G) \leq 15$ (see [2]). Furthermore, if $G$ has no 2-alternating cycles at all, then $M^{*}(G) \leq 13$ (see [2]), where both bounds are tight.

Douglas West asked for similar upper bounds on $M$-degrees of quasi-plane graphs without leaves and 4-faces. We proved in [8] that $M^{*}(G) \leq 15$ if $G$ is embedded into the plane or the projective plane and $M^{*}(G) \leq 18$ if $G$ is embedded into the torus or the Klein bottle. Both bounds are sharp.

Wang [16] proved that $M^{*}(G) \leq 6$ for every toroidal or Kleinian graph $G$ that has no leaves, 4-cycles, and 5-cycles. This bound is sharp.

The purpose of this paper is to prove Theorems 3 to 7 below:

Theorem 3. Let $G$ have no leaves and be either a projective-planar graph without 4- and 5-cycles, or be a toroidal or Kleinian graph without cycles of length from 4 to 6 . Then $M^{*}(G) \leq 5$. Both bounds on the length of forbidden cycles are sharp.

As explained in [13], this implies

Corollary 1. Let $G$ be either a projective-planar graph without 4- and 5-cycles, or a toroidal or Kleinian graph without cycles of length from 4 to 6 . Then

(i) $G$ has an edge-partition into a forest and a subgraph $H$ with $\Delta(H) \leq 4$;

(ii) the game chromatic number and the game coloring number of $G$ is at most 8.

Theorem 4. Let $G$ have no leaves and be either a projective-planar graph without cycles of length from 4 to 7 , or be a toroidal or Kleinian graph without cycles of length from 4 to 8 . Then $M^{*}(G) \leq 4$. This bound is attained even on planar graphs having no cycles of length from 4 to arbitrarily large number. Both bounds on the length of forbidden cycles are sharp.

This theorem and the next two theorems have corollaries similar to Corollary 1 on edge-partitions and game chromatic number of the corresponding graphs. We will not state them explicitly.

For $k \geq 4$, by the $k$-sunflower, $S_{k}$, we mean the graph obtained from $k$-cycle $C_{k}$ as follows: for every edge $x y$ add a new vertex $w=w(x y)$ and edges $w x$ and $w y$. For example, $S_{4}$ is depicted in Fig. 1 (left). This graph has $k$ vertices of degree 2 and $k$ vertices of degree 4 . Observe that $S_{k}$ has no cycles of length from 4 to $k-1$, while $M^{*}\left(S_{k}\right)=4$.

Therefore, to be able to find a graph with $M^{*}(G) \leq 3$, we consider graphs without 3-cycles sharing a vertex (without a butterfly). 

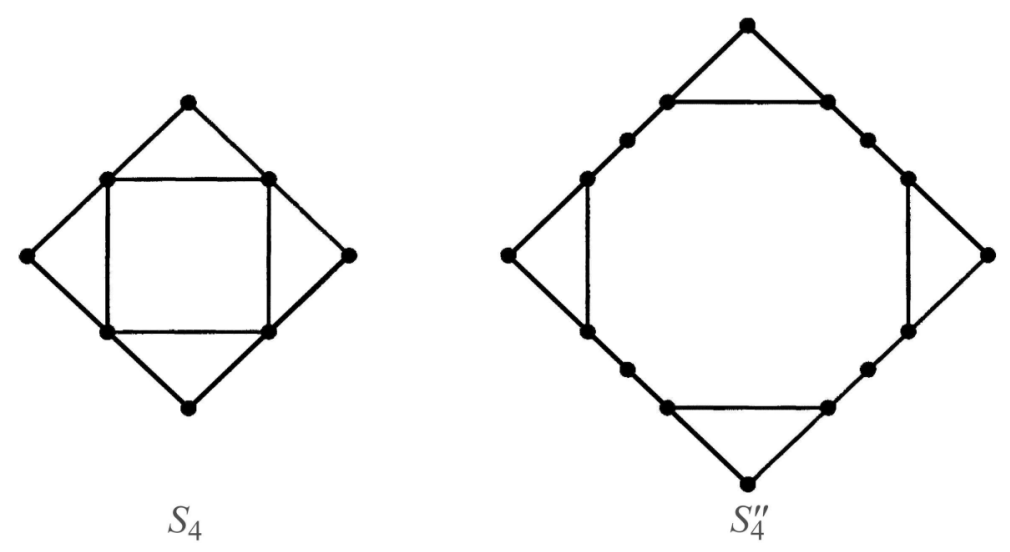

Fig. 1. Sunflower $S_{4}$ on the left and split sunflower $S_{4}^{\prime \prime}$ on the right.

Theorem 5. Suppose $G$ is a graph without butterflies and leaves. If $G$ is projective-planar and has no cycles of length from 4 to 9 , or if $G$ is a toroidal or Kleinian graph that has no cycles of length from 4 to 10 , then $M^{*}(G) \leq 3$. Both bounds are sharp.

Let $d_{T}(G)$ be the minimum distance between triangles in $G$. Saying that $d_{T}(G) \geq 1$ is another way to say that $G$ has no butterflies. Our next result is a sufficient condition for a graph to have $M^{*}(G)=2$. The following construction of split sunflowers says that condition $d_{T}(G) \geq 2$ is not sufficient: we take a sunflower $S_{k}$ and split each 4 -vertex into two vertices (of degree 3 ) joined by a path of length two as in Fig. 1 (right). The obtained graph $S_{k}^{\prime \prime}$ has $M^{*}\left(S_{k}^{\prime \prime}\right)=3$ and has no cycles of length $4 \leq l \leq 3 k-1$.

Theorem 6. Suppose $G$ is a graph with $\delta(G) \geq 2$ and $d_{T}(G) \geq 3$. If $G$ is projective-planar and has no cycles of length from 4 to 25 , or if $G$ is a toroidal or Kleinian graph that has no cycles of length from 4 to 30 , then $M^{*}(G)=2$. Both bounds are sharp.

Let $N(S)$ denote the Euler characteristics of a surface $S$. Recall that $N(S)=2$ if $S$ is the plane, $N(S)=1$ if $S$ is the projective plane, and $N(S)=0$ if $S$ is the torus or the Klein bottle; for the other surfaces $N(S)<0$.

In [9], we proved that any graph $G$ without 4 -cycles and leaves embedded into a surface $S$ with $N(S)<0$ and having more than $-72 N(S)$ edges has $M^{*}(G) \leq 8$.

From the proofs of Theorems 3-6, we will deduce the following.

Theorem 7. If a graph $G$ without leaves is embeddable into a surface $S$ with 
$N(S)<0$ and has more than $-2049 N(S)$ edges, then the following statements hold:

(i) if $G$ has no 4- and 5-cycles, then $M^{*}(G) \leq 6$;

(ii) if $G$ has no cycles of length from 4 to 6 , then $M^{*}(G) \leq 5$;

(iii) if $G$ has no cycles of length from 4 to 8 , then $M^{*}(G) \leq 4$;

(iv) if $G$ has no butterflies and cycles of length from 4 to 10 , then $M^{*}(G) \leq 3$;

(v) if $d_{T}(G) \geq 3$ and no cycles of length from 4 to 30 , then $M^{*}(G)=2$.

Thus, a large graph on a fixed surface $S$ with $N(S)<0$ behaves in terms of $M^{*}$ as a graph embeddable into the torus or the Klein bottle.

In the next section, we show that all restrictions on cycle lengths in Theorems 36 are sharp. In Sections 3 through 7, we prove Theorem 3 through Theorem 7 respectively.

\section{Extremal Examples}

\section{Examples for Theorem 3.}

In [9], an infinite family of quadrangle-free planar graphs $G$ with $M^{*}(G)=7$ is described. A construction of a toroidal or Kleinian graph without $C_{4}$ and $C_{5}$ with $M^{*}(G)=6$ is as follows: take a cubic graph $G^{\prime}$ of girth six (there are many such graphs embeddable into the torus or the Klein bottle) and for every edge $x y$ add a new vertex $w=w(x y)$ and edges $w x$ and $w y$. The degree of each "old" vertex in the new graph $G$ is six, and the "new" vertices form an independent set. Thus $M^{*}(G)=6$ and $G$ has neither 4-cycles nor 5-cycles.

\section{Examples for Theorem 4.}

First observe that the $k$-sunflower has no cycles of length from 4 to $k-1$, while $M^{*}\left(S_{k}\right)=4$.

Now we show that the bounds on the length of forbidden cycles are sharp. Consider the truncated icosahedron in Fig. 2. It has the property that each cycle that is not the boundary of a face has length at least 9 . Subdivide every edge of the bold matching of the graph $G^{\prime}$ in this figure with a vertex, and for every thin edge $x y$, add a vertex $w=w(x y)$ adjacent only to $x$ and $y$. Since bold edges form a matching in $G^{\prime}$, every vertex of $G$ has in the new graph $G$ degree exactly five. Since every 5 -face in $G^{\prime}$ contains two bold edges, and every 6 -face contains at least one edge, the new graph has no 4-, 5-, or 6-cycles.

A construction of a toroidal or Kleinian graph without $C_{4}-C_{7}$ with $M^{*}(G)=5$ is as follows. There are many cubic graphs $G^{\prime}$ that can be embedded into the torus or into the Klein bottle in such a way that (a) every face is a 6-face, (b) every cycle 


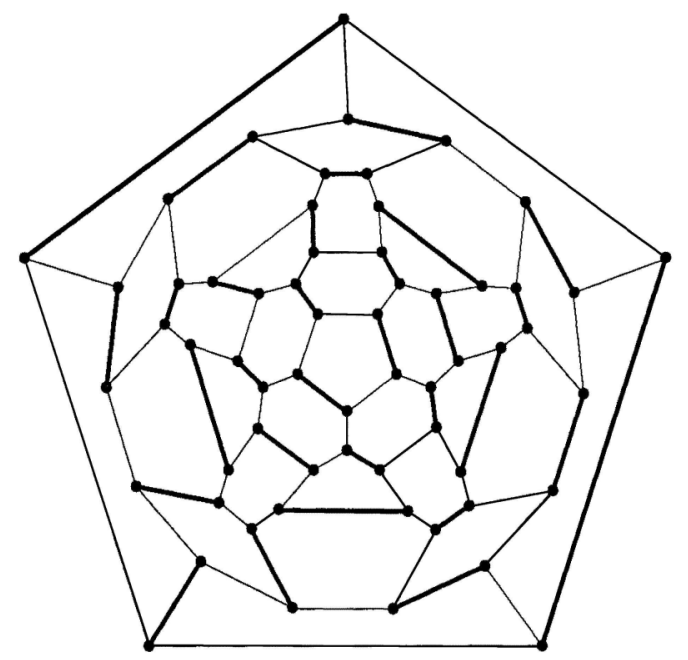

Fig. 2. A preparatory graph $G^{\prime}$ to get a planar graph $G$ with $M^{*}(G)=5$ and with no cycles of length from 4 to 6 .

that is not the boundary of a face has length at least 10 , and (c) there is a proper edge 3-coloring of $G^{\prime}$ such that in each 6 -face the opposite edges have the same color. Fix a color class $C$ in such an edge coloring. Subdivide each edge of $C$ with a vertex and for every edge $x y$ not in $C$, add a vertex $w=w(x y)$ adjacent only to $x$ and $y$. The new graph is our $G$. Again, the degree of every vertex of $G$ in $G$ is five. Since every face has two edges in $C$, our graph $G$ has no $k$-cycles for $4 \leq k \leq 7$.

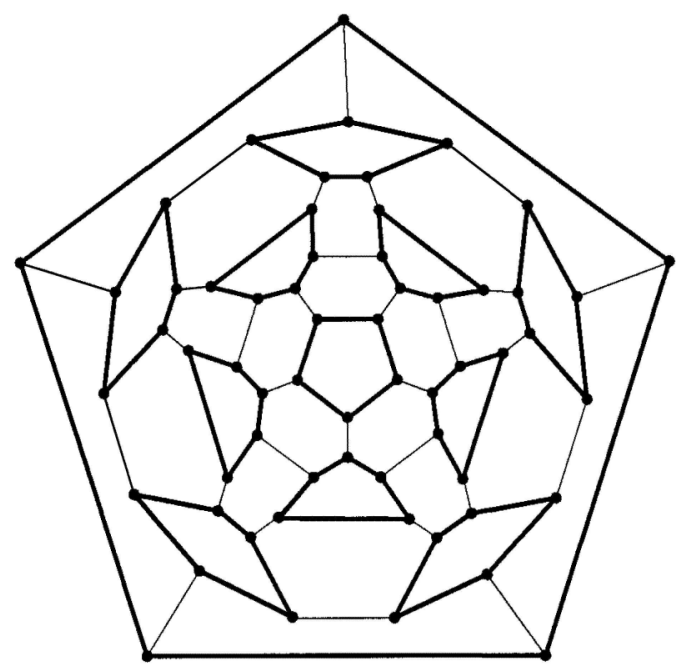

Fig. 3. A preparatory graph $G^{\prime}$ to get a planar graph $G$ with $M^{*}(G)=4$ and with no cycles of length from 4 to 8 . 


\section{Examples for Theorem 5.}

The basic graphs $G^{\prime}$ for constructing toroidal and Kleinian graphs $G$ without cycles of length from 4 to 9 having $\delta(G)=2$ and $M^{*}(G)=4$ are the same as in the previous paragraph, satisfying (a)-(c). This time, we subdivide the edges that are not in $C$ and for every edge $x y$ in $C$, add a vertex $w=w(x y)$ adjacent only to $x$ and $y$. Every vertex of $G$ becomes of degree four in $G$, and four edges of every face of $G^{\prime}$ are subdivided, by (c) our graphs $G$ have no $k$-cycles for $4 \leq k \leq 9$.

To construct a planar graph, we use the truncated icosahedron with a bold 2factor as in Fig. 3. As in the previous paragraph, subdivide every edge of the bold 2 -factor of the graph $G^{\prime}$ in this figure with a vertex, and for every thin edge $x y$, add a vertex $w=w(x y)$ adjacent only to $x$ and $y$. Since all edges of each 5-face and three edges of each 6-face in $G^{\prime}$ are subdivided, we get a planar graph $G$ with $\delta(G)=2$ and $M^{*}(G)=4$ that has no $k$-cycles for $4 \leq k \leq 8$.

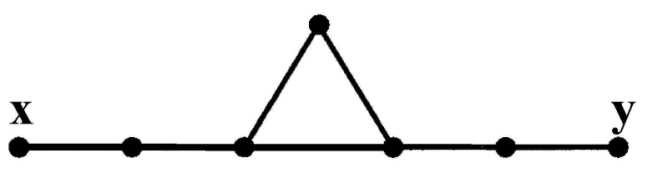

Fig. 4. A replacement for each edge in an auxiliary graph $G^{\prime}$ for Theorem 6 .

\section{Examples for Theorem 6.}

Let $G^{\prime}$ be either a cubic planar graph of girth $g\left(G^{\prime}\right)=5$ or a toroidal or Kleinian cubic graph of girth $g\left(G^{\prime}\right)=6$. We obtain $G$ by replacing each edge $x y$ with the graph in Fig. 4 (a path of length five with a triangle on the central edge). By construction, $M^{*}(G)=3$ and a shortest cycle that is not a triangle in $G$ has length $5 g\left(G^{\prime}\right)$.

\section{Proof of Theorem 3}

Let $G$ be a counterexample to Theorem 3 with the fewest vertices. Fix some embedding of $G$ and let $F(G)$ be the set of faces of this embedding. Let $d(y)$ denote the degree of a vertex $y$ or of a face $y$. Call a vertex $v$ senior, if $d(v) \geq 6$ and minor otherwise. By the choice of $G, M^{*}(G) \geq 6$, and hence

(1) Each closed walk $\left(w_{1}, \ldots, w_{t}\right)$ encounters at least $\left\lceil\frac{t}{2}\right\rceil$ senior vertices.

Claim 1. $G$ is connected.

Proof. Suppose that $G$ is the vertex-disjoint union of graphs $G_{1}$ and $G_{2}$, and there are no edges between $V\left(G_{1}\right)$ and $V\left(G_{2}\right)$. Note that $M^{*}(G)=\min \left\{M^{*}\left(G_{1}\right)\right.$, 
$\left.M^{*}\left(G_{2}\right)\right\}$, and thus one of $G_{1}$ or $G_{2}$ has fewer vertices than $G$ and yet has the same $M^{*}$ as $G$. This contradicts the minimality of $G$.

Since $G$ is connected, the boundary of every face is a closed walk.

In [9], we proved the following.

Claim 2. Suppose a graph has neither adjacent triangles nor adjacent vertices of degree 2. Then for every vertex $v$, the total number of triangular faces and 2 -vertices incident with $v$ is at most $d(v)$. 6.

We will use discharging to obtain a contradiction with the assumption $M^{*}(G) \geq$

Let the initial charge of every $x \in V(G) \cup F(G)$ be $\mu(x)=d(x)-4$.

By Euler's formula $|V(G)|-|E(G)|+|F(F)| \geq N(S)$, we have

$$
\sum_{x \in V(G) \cup F(G)}(d(x)-4)=\sum_{x \in V(G) \cup F(G)} \mu(x) \leq-4 N(S) .
$$

A 2-vertex on the boundary of a triangular face will be called triangular. The vertices and faces of $G$ discharge their initial charge by the following rules:

Rule 1. Every senior vertex gives $\frac{1}{3}$ to each adjacent 2 -vertex and each incident triangular face.

Rule 2. Every non-triangular face gives $\frac{1}{3}$ to each incident edge and $\frac{2}{3}$ to each incident triangular vertex of degree 2 .

Rule 3. Every edge incident with a minor vertex $v$ gives its charge to $v$. If both ends of edge $x y$ are senior vertices and $x y$ is on the boundary of a triangular face $f$, then $x y$ gives its charge to $f$.

Clearly, the resulting charge of every edge is nonnegative. It remains to show that the final charge $\mu^{*}(y)$ is nonnegative for each $y \in V(G) \cup F(G)$, and that the final charge of every face of length at least 7 is strictly positive. This yields a contradiction to (2), since the total charge does not change, and hence should be strictly negative when $G$ is projective-planar, and non-positive when $G$ is a toroidal or Kleinian graph. Recall that every quasiplane leafless graph $G$ with $M^{*}(G) \geq 5$ and no 4-cycles has non-triangular faces.

If $y$ is a triangular face, then $\mu^{*}(y) \geq 3-4+3 \times \frac{1}{3}=0$ by Rules 1 and 3 .

Suppose $y$ is a face with $d(y) \geq 6$. If $y$ is not incident with triangular 2-vertices, then $\mu^{*}(y) \geq d(y)-4-\frac{d(y)}{3}=\frac{2(d(y)-6)}{3}$. This is nonnegative when $d(y)=6$ and strictly positive when $d(y) \geq 7$. Now suppose $y$ is incident with $k \geq 1$ triangular 2 -vertices. We delete all $k$ vertices and as a result obtain the new face $y^{\prime}$ of size 
at least 6 in the case of projective planar graphs and at least 7 otherwise. Observe that $d(y)=d\left(y^{\prime}\right)+k$ and, on the other hand, the expenditure of $y$ is also precisely by $k$ greater than that of $y^{\prime}$. Indeed, every triangular 2 -vertex contributes 1 to the size of $y$ and increases its expenditure by $2 \times \frac{1}{3}-\frac{1}{3}+\frac{2}{3}=1$ by Rule 2 .

Suppose now that $y$ is a vertex.

If $d(y)=2$, then by Rule 1 it gets $\frac{2}{3}$ from the adjacent vertices and exactly $\frac{4}{3}$ more: either all from the incident edges, if $y$ has no incident triangular faces, or $\frac{2}{3}$ from the incident edges and $\frac{2}{3}$ from the incident non-triangular face by Rules 2 and 3. Hence, $\mu^{*}(y)=0$.

Let $d(y)=3$. Since $G$ has no 4 -cycles and hence at most one face incident with $y$ is a triangle, by Rule 3, $y$ gets at least $\frac{4}{3}$ from the incident edges. Hence, $\mu^{*}(y)>0$.

If $4 \leq d(y) \leq 5$, then $v$ gives out nothing, and $\mu^{*}(y)=d(y)-4 \geq 0$.

If $d(y) \geq 6$, then $\mu^{*}(y) \geq d(y)-4-\frac{d(y)}{3} \geq 0$ by Claim 2 .

This completes the proof of Theorem 3 .

\section{Proof of Theorem 4}

Let $G$ be a counterexample to the main statement of Theorem 4. This time, call a vertex $v$ senior, if $d(v) \geq 5$ and minor otherwise. As in the previous section, let the initial charge of every $x \in V(G) \cup F(G)$ be $\mu(x)=d(x)-4$.

The vertices and faces of $G$ discharge their initial charge by the following rules:

Rule 1. Every senior vertex gives $\frac{1}{2}$ to each incident triangular face.

Rule 2. Every non-triangular face gives $\frac{1}{2}$ to each incident edge and $\frac{1}{2}$ to each incident triangular 2-vertex.

Rule 3. Every edge incident with a minor vertex $v$ gives its charge to $v$. If both ends of edge $x y$ are senior vertices and $x y$ is on the boundary of a triangular face $f$ whose third vertex is a 2-vertex, then $x y$ gives its charge to this 2 -vertex.

Clearly, every edge has a nonnegative resulting charge. It remains to show that the final charge $\mu^{*}(y)$ is nonnegative for each $y \in V(G) \cup F(G)$, and that the final charge of every face of length at least 9 is strictly positive. This yields a contradiction to (2), as in the proof of Theorem 3. Recall that every quasiplane graph $G$ with $M^{*}(G) \geq 5$ and no 4 -cycles has non-triangular faces.

If $y$ is a triangular face, then $\mu^{*}(y) \geq 3-4+2 \times \frac{1}{2}=0$ by Rule 1 .

Suppose $y$ is a face with $d(y) \geq 8$. If $y$ is not incident with triangular 2 -vertices, then $\mu^{*}(y) \geq d(y)-4-\frac{d(y)}{2}=\frac{d(y)-8}{2}$. This is strictly positive when $d(y) \geq 9$. 
Now suppose $y$ is incident with $k \geq 1$ triangular 2-vertices. When we delete all $k$ vertices, we obtain a new face $y^{\prime}$ of size at least 8 in the case of projective planar graphs and at least 9 , otherwise. Observe that $d(y)=d\left(y^{\prime}\right)+k$ and, on the other hand, the expenditure of $y$ is also precisely by $k$ greater than that of $y^{\prime}$. Indeed, every triangular 2-vertex contributes 1 to the size of $y$ and increases its expenditure by $\frac{1}{2}-\frac{1}{2}+2 \times \frac{1}{2}=1$ by Rule 2 .

Now let $y$ be a vertex.

First suppose $d(y)=2$. If $y$ is not triangular, then by Rule 2 each of the incident edges gets 1 from the two incident faces, and by Rule $3 y$ gets $2 \times 1$ from these edges. If $y$ is triangular, then by Rule 3 it gets $2 \times \frac{1}{2}$ from the incident edges and $\frac{1}{2}$ from the opposite edge on the incident triangular face. Apart from it, by Rule 2, $y$ gets $\frac{1}{2}$ from the incident non-triangular face. Hence, $\mu^{*}(y)=0$.

If $d(y)=3$, then since $G$ has no 4-cycles, by Rule 3, it gets at least 2 from the incident edges. Hence, $\mu^{*}(y)>0$.

If $d(y)=4$, then $v$ gives out nothing, and $\mu^{*}(y)=d(y)-4=0$.

If $d(y)=5$, then $\mu^{*}(y) \geq 5-4-2 \times \frac{1}{2}=0$ by Rule 1 .

If $d(y) \geq 6$, then $\mu^{*}(y) \geq d(y)-4-\frac{d(y)}{4} \geq 0$ by Rule 1 .

This completes the proof of Theorem 4 .

\section{Proof of Theorem 5}

Let $G^{\prime}$ be a counterexample to the main statement of Theorem 5. Now call a vertex $v$ senior, if $d(v) \geq 4$ and minor otherwise.

Let $G$ be obtained from $G^{\prime}$ by subdividing with a vertex every edge $x y$ that does not belong to any 3-cycle and is incident only with senior vertices. By construction, $M^{*}(G) \geq 4$ and no cycles of intermediate length arise. Thus, $G$ is also a counterexample to the main statement of Theorem 5 , and has the additional property that

(3) senior vertices are adjacent only if they belong to a common triangle.

As in the two previous proofs, let the initial charge of every $x \in V(G) \cup F(G)$ be $\mu(x)=d(x)-4$. The vertices and faces of $G$ discharge their initial charge in two stages. First, every non-triangular face gives 1 to every incident edge that is also incident with a triangle face, and $\frac{1}{2}$ to every other edge. The resulting charge of every edge becomes exactly 1 . The next claim is crucial.

Claim 3. The new charge $\mu^{\prime}(y)$ is nonnegative for every non-triangular face. Furthermore, if $G$ has no 10-cycles, then the new charge of some non-triangular face is positive. 
Let $f$ be a face of length $l \geq 10$. An edge incident with $f$ is $f$-easy if it gets $\frac{1}{2}$ from $f$ and is $f$-tough if it gets 1 from $f$. By (3), the $f$-easy edges can be partitioned into pairs such that each pair has a common end that is a minor vertex. If we have at least four such pairs, then the first half of the claim is proved. Suppose that there are at most three such pairs. Deleting these at most six edges breaks the remaining at least $l-6$ edges into some $j \leq 3$ intervals of $f$-tough edges. By (3), each such interval has at most two edges. Moreover, if an interval has two edges $x_{i-1} x_{i}$ and $x_{i} x_{i+1}$, then $x_{i-1} x_{i+1}$ is also an edge in $G$. Thus if exactly $h$ of the intervals have length two, then $l \geq 10+h$. Therefore, $j+h \geq(10+h)-6$, a contradiction to $j \leq 3$.

To prove the second statement of the claim, suppose that $G$ has no cycles of length $k$ for $4 \leq k \leq 10$. Suppose that each non-triangular face $f$ has exactly eight $f$-easy edges. Let $f_{0}$ be a face of length $l \geq 11$. By the same argument as in the previous paragraph, after deleting $f_{0}$-easy edges from the boundary of $f_{0}$, the remaining edges break into at least 3 intervals. This means that some interval of $f_{0}$-easy edges on the boundary of $f_{0}$ consists of exactly two edges, say $v_{1} v_{2}$ and $v_{2} v_{3}$. This, in turn, means that edges $v_{l} v_{1}$ and $v_{3} v_{4}$ belong to triangular faces, and vertices $v_{1}$ and $v_{3}$ are senior. Let $f_{1}$ be the face that shares edges $v_{1} v_{2}$ and $v_{2} v_{3}$ with $f_{0}$. Since $G$ has no butterflies, the edge incident with $f_{1}$ containing $v_{1}$ and other than $v_{1} v_{2}$ does not belong to a triangular face, and hence is $f_{1}$-easy. Similarly, the edge incident with $f_{1}$ containing $v_{3}$ and other than $v_{2} v_{3}$ also is $f_{1}$-easy. By (3), $f_{1}$ contains at least six consecutive $f_{1}$-easy edges. Then the new charge of $f_{1}$ is positive. This proves the claim.

At the second stage of the discharging, each edge incident with a minor vertex gives all its charge (equal to 1 ) to this minor vertex. Each edge not incident with a minor vertex gives all its charge to the incident triangular face.

Note that each triangular face gets at least 1 and each minor vertex at least 2 . Senior vertices and non-triangular faces do not change their charge at this stage. This completes the proof of Theorem 5.

\section{Proof of Theorem 6}

Let $G$ be a counterexample to the main statement of the theorem. In particular, $M^{*}(G) \geq 3$ and hence

(4) each path connecting any two triangular faces has an internal $\geq 3$-vertex.

Let $G^{\prime}$ be obtained from $G$ by contracting every triangular face of $G$ into a vertex. Since each 3 -cycle can have at most one 2 -vertex, $\delta(G) \geq 2$. Then we replace each inclusion maximal path in $G^{\prime}$ whose internal vertices all have degree 2 with a single edge to obtain a graph $G^{\prime \prime}$ with $\delta\left(G^{\prime \prime}\right) \geq 3$. 
Every graph $G^{\prime \prime}$ with $\delta\left(G^{\prime \prime}\right) \geq 3$ embedded into the plane or projective plane has a face of size at most 5 , and every such graph embedded into the torus or the Klein bottle has a face of size at most 6 . Let such a face be $f^{\prime \prime}=v_{1} \ldots v_{k}$.

Let $f$ be the pre-image of $f^{\prime \prime}$ in graph $G$ embedded into the corresponding surface. Since $G$ has no adjacent 2 -vertices, our $f$ is not a triangular face (because all triangular faces of $G$ were contracted) and hence has size at least 26 when $G$ is projective-plane and 31 when $G$ is embedded into the torus or the Klein bottle. By (4), the pre-image of each edge of $f^{\prime \prime}$ contains at most one edge of a triangular face, and hence contains at most five edges in total. This contradicts our choice of $f^{\prime \prime}$.

This contradiction completes the proof of Theorem 6 .

\section{Proof of Theorem 7}

We give a proof only for the most difficult part (iv) of Theorem 7, because the argument for other cases is simpler.

If $G$ has no butterflies and cycles of length from 4 to 10, then as follows from the proof of Theorem 5, all vertices, edges and faces have nonnegative new charges $\mu^{*}$. Moreover, if $f$ is a nontriangular face with $\mu^{*}(f)=0$, then $d(f) \leq 16$. Since in the discharging in Theorem 5 we deal only with charges that are multiples of $\frac{1}{2}$, it follows that $\mu^{*}(f) \geq \frac{1}{2}$ unless $\mu^{*}(f)=0$. Furthermore, it is not hard to check that $\mu^{*}(f) \geq k-4$, provided that $d(f) \geq 4 k-3$, when $k \geq 5$.

Since $G$ is embedded in $S$, we have

$$
\sum_{x \in V \cup E \cup F} \mu^{*}(x)=\sum_{x \in V \cup F} \mu(x)=\sum_{x \in V \cup F}(d(x)-4)=-4 N(S) .
$$

Recall that $\mu^{*}(e)=0$ for every edge of $G, \mu^{*}(v) \geq 0$ for every $v \in V$, and $\mu^{*}(T)=0$ for every triangle $T$ in $G$. This implies that

$$
\sum_{f \in F, f \neq T} \mu^{*}(f) \leq-4 N(S)
$$

We want to redistribute the charges of the nontriangular faces of $G$ to the edges of $G$ so that every edge has charge at least $2^{-9}$. (This will give the result).

Note that every edge, $e$, is incident with a nontriangular face, $f_{0}$. However, $f_{0}$ may have $\mu^{*}\left(f_{0}\right)=0$. As mentioned in the course of the proof of Theorem 5, such $f_{0}$ is adjacent to a face $f^{+}$with $\mu^{*}\left(f^{+}\right)>0$. In this situation we say that $f_{0}$ is dependent on $f^{+}$. Our redistribution consists in dividing $\mu^{*}\left(f^{+}\right)$evenly among the edges incident with $f^{+}$and all faces dependent on $f^{+}$.

Clearly, $\mu^{*}\left(f^{+}\right)$is divided into at most $16 d\left(f^{*}\right)$ portions, $p$. If $d\left(f^{*}\right) \leq 16$ then $p \geq \frac{1}{2} \times \frac{1}{16} \times \frac{1}{16}=2^{-9}$; if $d(f) \geq 4 k-3$, where $k \geq 5$, then $p \geq(k-4) \times \frac{1}{4 k} \times \frac{1}{16} \geq$ 
$1 \times \frac{1}{20} \times \frac{1}{16}>2^{-9}$. Hence, for each edge $e$, its final charge, $\mu^{* *}(e)$ is at least $2^{-9}$. It follows that $|E| \geq-4 N(S) \times 2^{-9}$, a contradiction.

\section{REFERENCES}

1. H. L. Bodlaender, On the complexity of some coloring games, Intern. J. Found. Comput. Sci., 2 (1991), 133-147.

2. O. V. Borodin, Consistent colorings of graphs in the plane, Diskret. Analiz, 45 (1987), 21-27 (in Russian).

3. O. V. Borodin, On the total coloring of planar graphs, J. Reine Angew. Math., 394 (1989), 180-185.

4. O. V. Borodin, A generalization of Kotzig's theorem and prescribed edge coloring of plane graphs. Math. Notes Acad. Sci. USSR, 48 (1990), 1186-1190.

5. O. V. Borodin, Structural properties of plane graphs without adjacent triangles and an application to 3-colorings, J. of Graph Theory, 21 (1996), 183-186.

6. O. V. Borodin, A. N. Glebov, A. O. Ivanova, T. K. Neustroeva and V. A. Tashkinov, Sufficient conditions for planar graphs to be 2-distance $(\Delta+1)$-colourable, Siberian Electronic Math. Reports (http://semr.math.nsc.ru/), 1 (2004), 129-141 (in Russian).

7. O. V. Borodin, A. O. Ivanova and T. K. Neustroeva, Sufficient conditions for planar graphs with girth 6 to be 2-distance colourable, Siberian Electronic Math. Reports (http://semr.math.nsc.ru), 3 (2006), 441-450 (in Russian).

8. O. V. Borodin, A. O. Ivanova, A. V. Kostochka and N. N. Sheikh, Minimax degrees of quasiplane graphs without 4-faces, Siberian Electronic Math. Reports (http://semr.math.nsc.ru/), 4 (2007), 435-439.

9. O. V. Borodin, A. V. Kostochka, N. N. Sheikh, and G. Yu, $M$-degrees of quadranglefree planar graphs (submitted).

10. O. V. Borodin, A. V. Kostochka and D. R. Woodall, List edge and list total colourings of multigraphs, J. Combin. Theory (B) 71, 2 (1997), 184-204.

11. U. Faigle, U. Kern, H. A. Kierstead and W. T. Trotter, On the game chromatic number of some classes of graphs, Ars. Combin., 35 (1993), 143-150.

12. B. Grünbaum, New views on some old questions of combinatorial geometry, Colloquio Internazionale sulle Teorie Combinatorie, Vol. I. Rome: Accad. Nat. Lincei. 1976. 451-468 (Atti dei Convegni Lincei.; Vol. 17).

13. W. He, X. Hou, K. W. Lih, J. Shao, W. Wang and X. Zhu, Edge-partitions of planar graphs and their game coloring numbers, J. Graph Theory, 41 (2002), 307-317.

14. A. Kotzig, Contribution to the theory of eulerian polyhedra, Mat. Čas., 5 (1955), 101-103.

15. A. Kotzig, Contribution to the theory of Eulerian polyedra, Mat. Čas., 13 (1963), 20-34 (in Russian). 
16. W. Wang, Edge-partitions of graphs of nonnegative characteristic and their game coloring number. Discrete Math., 2 (2006), 262- 270.

17. P. Wernicke, Uber den Kartographischen Vierfarbensatz, Math. Ann., 58 (1904), 413-426.

18. J. L. Wu, On the linear arboricity of planar graphs of nonnegative characteristic and their game coloring number, J. Graph Theory, 31 (1999), 129-134.

Oleg V. Borodin

Sobolev Institute of Mathematics,

Novosibirsk 630090,

Russia

E-mail: brdnoleg@math.nsc.ru

Anna O. Ivanova

Yakutsk State University,

Yakutsk 677000,

Russia

E-mail: shmgnanna@mail.ru

Alexandr V. Kostochka

Department of Mathematics,

University of Illinois,

Urbana, IL 61801,

U.S.A.

and

Sobolev Institute of Mathematics,

Novosibirsk 630090,

Russia

E-mail: kostochk@math.uiuc.edu.

Naeem N. Sheikh

Department of Mathematics,

University of Illinois,

Urbana, IL 61801,

U.S.A.

E-mail: nsheikh@math.uiuc.edu 